\title{
The Diagnostic Value of Urinary Secretory Antigen Target of 6 kDa in Childhood Pulmonary Tuberculosis
}

\author{
Agustin Iskandar ${ }^{1 *}$ (D), Ella Melissa Lawanto ${ }^{1}$ (D), Maimun Zulhaidah ${ }^{1}$ (D), Ery Olivianto ${ }^{2}$, Kusworini Handono ${ }^{1}$, \\ Muhammad Anshory ${ }^{3}$ iD, Andrea Aprilia ${ }^{1}$ iD, Aryati Aryati $^{4}$ (D) \\ ${ }^{1}$ Department of Clinical Pathology and Parasitology, Faculty of Medicine, Universitas Brawijaya, Malang, Indonesia; ${ }^{2}$ Department \\ of Child Health, Faculty of Medicine, Universitas Brawijaya, Malang, Indonesia; ${ }^{3}$ Department of Internal Medicine, Faculty \\ of Medicine, Universitas Brawijaya, Malang, Indonesia; ${ }^{4}$ Department of Clinical Pathology, Faculty of Medicine, Airlangga \\ University, Surabaya, Indonesia
}

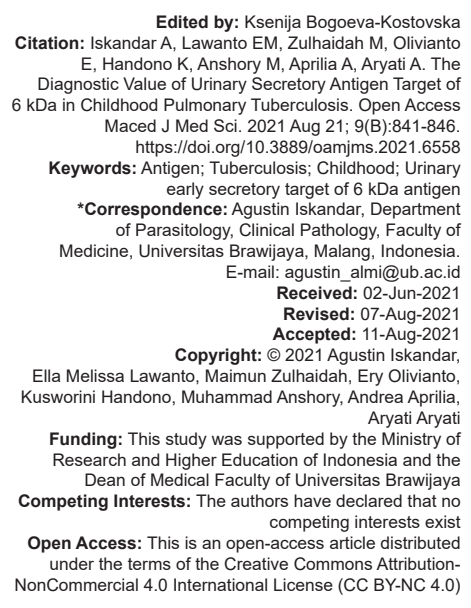

\section{Introduction}

Tuberculosis (TB) is one of the most prevalence infections worldwide and remained the most common cause of death from a single infection in 2019. The most prevalence region with TB infection is South-East Asia $(44 \%)$ and the prevalence in Indonesia is $8.5 \%$ [1]. The worldwide TB case load in children is not completely widely known due to the lack of diagnostic tools for these subjects and inadequate system for recording and reporting cases of childhood TB. The diagnosis of childhood TB is very difficult, often inaccurate, and can result in over or underdiagnosis, leading as consequence to inadequate treatment [2]. In infants and children, the inability or difficulty to expectorate the sputum, the lower bacillary load than can cause falsenegative results in staining (acid-fast stain) and culture [3]. Culture represents the gold standard examination of adult TB diagnosis but with a poor sensitivity (30-40\%) in children [4], [5]. Urine samples for diagnosis of TB have various advantages, especially in children, while it is a noninvasive tool that could be useful also in the case of extra-pulmonary TB and can be used in the contemporary presence of HIV infection which is characterized by fewer amount of bacteria in sputum [6]. Moreover, the WHO in 2011 banned the use of serological tests for the detection of antibodies against mycobacteria, since commercial serological tests provide inconsistent and imprecise findings for the detection of TB. Furthermore, the $\mathrm{WHO}$ also stated that until now there is no serology test viable that can be used for diagnosis of childhood TB and encourages further research in developing new TB serology tests for children based on antigen marker [7].

Early secretory target of $6 \mathrm{kDa}$ antigen (ESAT-6) was known to play an important role in TB bacterial virulence. ESAT-6 can cause cytolysis of the membrane and thus the evasion of MTB inside 
the phagosome [8]. ESAT-6 is a small protein for about $6 \mathrm{kDa}$ and could be unrestrictedly filtered by the glomerulus and be detected in the urine [9]. The study about in-vitro assays that measure interferon- $\gamma$ (IFN- $\gamma$ ) or IFN- $\gamma$ secreting T cells indicate that ESAT-6 is a potential diagnostic reagent that highly specific for active TB and is often detected during early infection in TB disease [10].

Mukundan et al. (2012) demonstrated the direct detection of ESAT-6, measured by ELISA, in serum samples of patients with active TB showing that ESAT-6 was detected in three samples of active TB but not in non-TB control samples [11]. Other study by Song et al. (2014) examined the diagnostic tests of cerebrospinal ESAT-6 with indirect ELISA method on meningitis TB, obtained a sensitivity of $88 \%$ and specificity of $92 \%$ [12].

So far there is no data have been reported on the value of urinary ESAT- 6 testing for diagnosis of TB both in adults and in children. The aim of our study was to evaluate the diagnostic value of urinary ESAT- 6 in childhood TB.

\section{Materials and Methods}

\section{Study design and patient population}

Between June 2018 and June 2019, a crosssectional study, with consecutive sampling collection, was conducted at Department of Child Health, Saiful

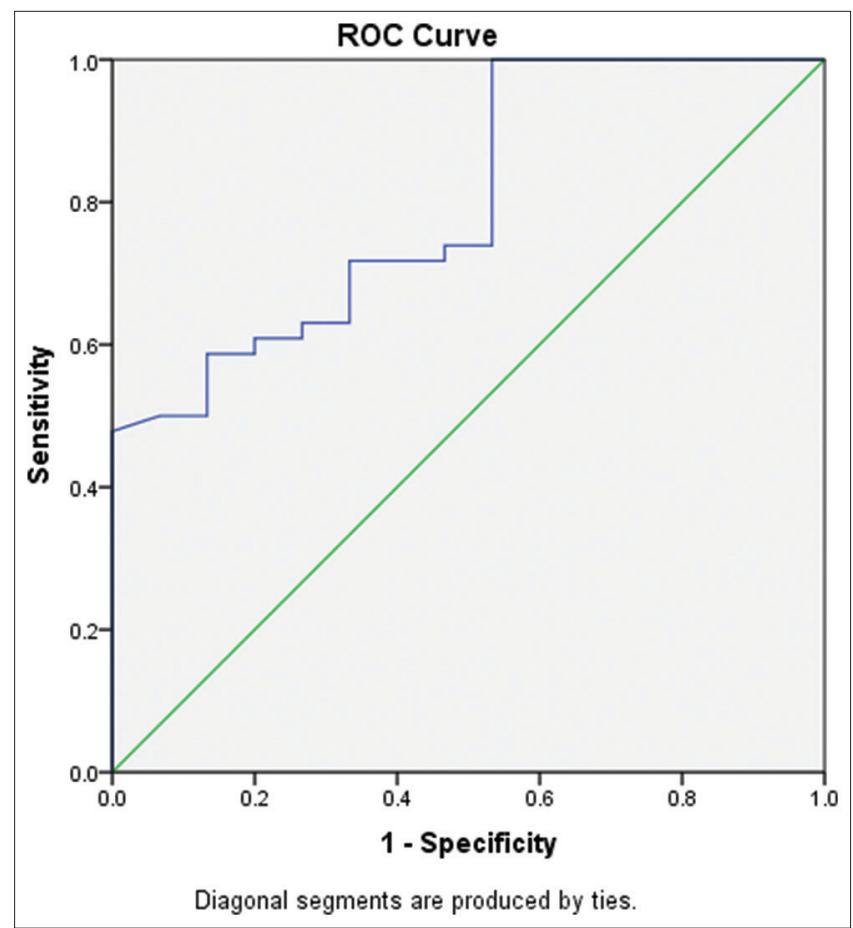

Figure 1: Area under the curve of this test was 0.8 (95\% Cl 0.68-0.92) by using receiver operating characteristic curve based on clinically diagnosed tuberculosis
Anwar General Hospital in Malang, Indonesia. The study population was consisted of 78 children aging 0-1 4 years old with clinical suspicion of pulmonary TB. The study was approved by the ethical commitee of the Faculty of Medicine Universitas Brawijaya/Saiful Anwar Hospital. Informed consent was obtained from either parent or relatives accompanying the patients.

Children aged 0-14 years suffered from cough more than 2 weeks, fever without clear ethiology, loss of body weight or poor weight gain, fatigue, malaise, and have history of contact with positive sputum smear adult TB patient were enrolled into the study. Subjects previously diagnosed to have pulmonary or extrapulmonary TB, with symptoms and sign of extrapulmonary TB, such as chronic cervical, submandibular, and supraclavicular lymph node enlargement, spine angulation, joint swelling and had already received anti-TB medications for more than 2 weeks were excluded. Subjects were also excluded if there were leukocyturia, hematuria, and moderatesevere proteinuria reflecting renal abnormalities found in urinalysis.

History taking was also aimed to check if there was the presence of household adult contact with active TB and previous history of anti-TB medication. Physical examination was performed systematically including evaluation of nutritional status using maternal and child health book (Indonesian Ministry of Health) for children aged $<5$ years and the CDC 2000 curve for children aged $>5$ years [2]. Chest $X$-ray was interpreted by senior pediatric pulmonologist (MSC). Interpretation would be suggestive of TB or not. However, the result of chest $X$-ray would be considered in relation to overall clinical presentation. Tuberculin skin tests were performed in all subjects using 2 TU PPD RT-23, intradermally applaied (Tuberculin skin test are considered positive if diameter of induration $\geq 10 \mathrm{~mm}$ ) [13]. Urine sample $( \pm 10 \mathrm{~mL})$ was taken at the moment of subjects in hospital presentation and urinary ESAT-6 level was measured using the ELISA method (Bioassay Technology Laboratory) [14]. Sputum samples were obtained by sputum induction or gastric lavage while sputum examination for acidfast staining (Ziehl-Neelsen) and culture (Lowenstein Jensen) was also performed.

Subjects were diagnosed as pulmonary TB and considered microbiologically confirmed when at least one positive result of sputum staining or positive MTB culture. If microbiology examination showed negative results, the subjects were considered as clinically confirmed TB when they defined as active TB by a pediatrician and has been designated to receive TB treatment and met at least two of the followings symptoms and signs suggestive of pulmonary TB; chest radiograph consistent with $\mathrm{TB}$; close contact of $\mathrm{TB}$ or positive tuberculin skin test; and noted positive clinical response after 2 months course of anti TB treatment. Subjects who met the inclusion criteria but unconfirmed by clinical and microbiological were considered as non-TB group (Flowchart 1). 


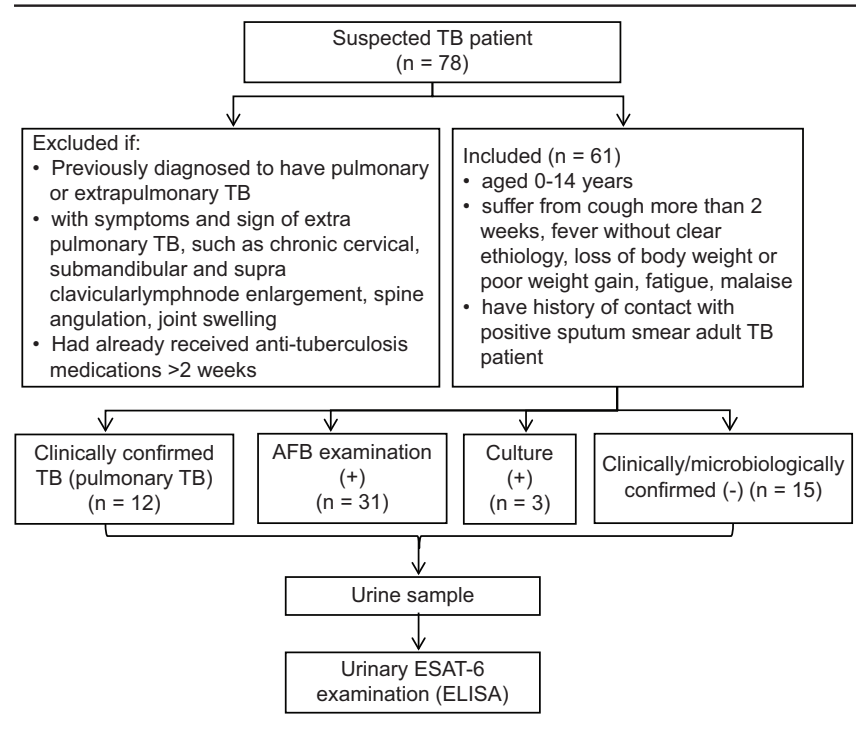

Flowchart 1: The flowchart of the study

\section{Statistical analysis} formula [15]:

Sample size determined using the following

$$
\mathrm{N}=\frac{\left(\mathrm{Z}_{\alpha} \sqrt{2} \mathrm{~V}_{1}+\mathrm{Z}_{\beta} \sqrt{ } 2 \mathrm{~V}_{1}+\mathrm{V}_{2}\right)}{\left(A U \mathrm{C}_{1}-\mathrm{AUC}_{2}\right)^{2}}
$$

The urine ESAT- 6 levels were evaluated as mean \pm SD. To determine the differences in the levels of ESAT- 6 in the 2 groups: (1) TB group and (2) non-TB group; the Mann-Whitney test or t-test were used with significance reached if $p$-value was at least $<0.05$. Receiver operating characteristic (ROC) curve plotted various intersection points for sensitivity and specificity values to determine the cut-off values. Positive predictive value (PPV), negative predictive value (NPV), positive likelihood ratio (PLR), and negative likelihood ratio (NLR) was calculated from $2 \times 2$ table analysis.

\section{Results}

Forty-six $(75 \%)$ of 61 subjects suspected of TB were eventually diagnosed with TB and $15(25 \%)$ were not. Of those diagnosed with TB, 34 (74 \%) were microbiologically confirmed cases either by sputum microscopy (91\%) or culture (9\%), and $12(26 \%)$ subjects were clinicaly confirmed TB. The characteristics of the subjects were shown in Table 1.

Urinary ESAT-6 level was higher in subjects with TB $(4.855 \pm 6.714) \mathrm{ng} / \mathrm{mL}$ compared to non-TB group $(1.503 \pm 0.946) \mathrm{ng} / \mathrm{mL}$ and statistically significant using Mann-Whitney test $(p=0.001)$. Using SPSS 20.0 software, cut-off value of urinary ESAT-6 level
Table 1: Characteristics of subjects

\begin{tabular}{lll}
\hline Characteristics & Non-TB $(\mathrm{n}=15)$ & TB $(\mathrm{n}=46)$ \\
\hline Age (year) & $2.04 \pm 2.91$ & $6.98 \pm 4.32$ \\
$0-1$ & $10(66.7)$ & $2(4.3)$ \\
$>1-5$ & $3(20.0)$ & $16(34.8)$ \\
$>5-14$ & $2(13.3)$ & $28(60.9)$ \\
Male sex & $10(66.7)$ & $30(65.2)$ \\
Positive Tuberculin skin test & 0 & $26(56.5)$ \\
Positive microbiology test & 0 & $34(73.9)$ \\
Kultur $(+)$ & 0 & $3(6.5)$ \\
BTA $(+)$ & 0 & $31(67.4)$ \\
Urinary LAM $(\mathrm{ng} / \mathrm{mL})$ & $1.503 \pm 0.946$ & $4.855 \pm 6.714$ \\
Nutritional status & & \\
$\quad$ Normal & $7(46.7)$ & $18(39.1)$ \\
$\quad$ Malnourished & $8(53.3)$ & $28(60.9)$ \\
\hline Data was presented in $\mathrm{n}(\%)$, and mean \pm SD, TB: Tuberculosis.
\end{tabular}

was $1.91 \mathrm{ng} / \mathrm{mL}$ when compared to diagnosis of $T B$ (including clinically and microbiology confirmed cases). When only positivity of microbiology examination used as standard reference, cut off value of urinary ESAT-6 level was $2.45 \mathrm{ng} / \mathrm{mL}$.

Table 2 showed the number of subjects based on the value of the cut-off ESAT- 6 and diagnosis of TB. Based on this $2 \times 2$ crosstable, the sensitivity of the test was $72 \%$, specificity $67 \%$, PPV $87 \%$, NPV $43 \%$, PLR 2.18, and NLR 0.42 .

Table 2: Urinary ESAT-6 level (cut off point $1.91 \mathrm{ng} / \mathrm{mL}$ ) and diagnosis of TB $2 \times 2$ crosstable

\begin{tabular}{llll}
\hline Urinary ESAT-6 & \multicolumn{2}{l}{ Diagnosis } & Total \\
\cline { 2 - 3 } & TB & Non-TB & \\
\hline ESAT-6 $\geq 1.91 \mathrm{ng} / \mathrm{mL}$ & 33 & 5 & 38 \\
ESAT- $6<1.91 \mathrm{ng} / \mathrm{mL}$ & 13 & 10 & 23 \\
Total & 46 & 15 & 61 \\
\hline ESAT: Early secretory target of $6 \mathrm{kDa}$ antigen, TB: Tuberculosis. & &
\end{tabular}

Table 3 showed the number of subjects based on the value of the cut-off ESAT- 6 and microbiological confirmed (acid-fast staining or culture). Based on this $2 \times 2$ crosstable, the sensitivity of the test was $65 \%$, specificity $67 \%$, PPV $71 \%$, NPV $60 \%$, PLR 1.97 , and NLR 0.52 .

Table 3: Urinary ESAT-6 level (cut off point $2.45 \mathrm{ng} / \mathrm{mL}$ ) and microbiological confirmed $2 \times 2$ crosstable

\begin{tabular}{llll}
\hline Urinary ESAT-6 & Microbiological confirmed & Total \\
\cline { 2 - 3 } & Acid fast stain/culture (+) & Acid fast stain/culture (-) & \\
\hline ESAT-6 $\geq 2.45 \mathrm{ng} / \mathrm{mL}$ & 22 & 9 & 31 \\
ESAT-6 $<2.45 \mathrm{ng} / \mathrm{mL}$ & 12 & 18 & 30 \\
Total & 34 & 27 & 61 \\
\hline ESAT: Early secretory target of $6 \mathrm{kDa}$ antigen. & &
\end{tabular}

ROC curve analysis showed area under the curves (AUCs) of $0.8(95 \% \mathrm{Cl} 0.68-0.92)$ to the clinical diagnosis of childhood TB (Figure 1), and when we analyze by using microbiological confirmation as reference standard, the AUCs was $0.71(95 \% \mathrm{Cl} 0.58$ 0.84 ) as shown in Figure 2.

\section{Discussion}

In order to provide a definitive diagnosis of TB is mandatory to find MTB on microbiological examination, including direct microscopic examination of smear or tissue biopsy and culture examination. However, the 


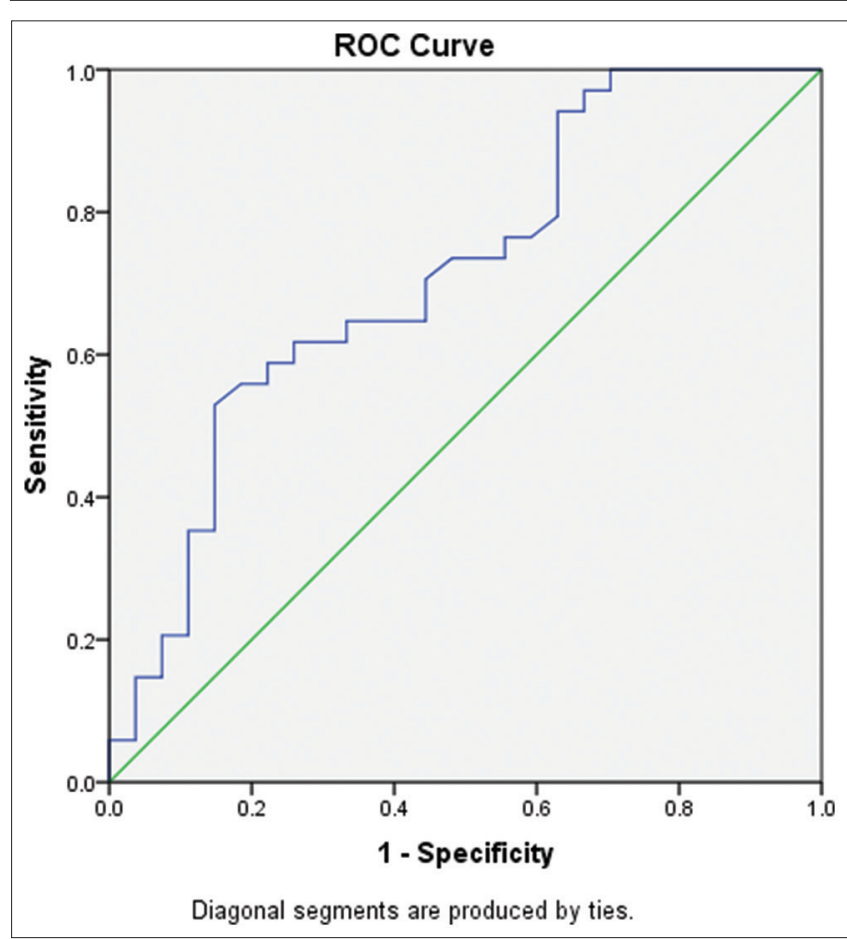

Figure 2: Receiver operating characteristic curve showed area under the curve of this test was $0.71(95 \% \mathrm{Cl} 0.58-0.84)$ by using microbiological confirmed as reference standard

diagnosis of childhood TB presents many caveats, such as (1) TB can mimic many common childhood diseases, including pneumonia, generalized bacterial and viral infections, (2) the absence of a practical reference test or gold standard, (3) the inability of pediatric patients to provide sputum, (4) A nonspecific clinical presentation (up to $50 \%$ of children may be asymptomatic in early stages of the disease), (5) the lower bacillary load often smear negative [16]. The number of MTB in the bronchial secretions of children is fewer than adults because of the location of tissue damage in primary pulmonary is located in the hilar lymph nodes and peripheral lung parenchyma and the severity of lung damage is not as severe as in adult patients [3]. (6) Confirmation by culture of MTB, the gold standard of diagnosis in adult TB, rarely exceeds $30-40 \%$ sensitivity.

As consequence the diagnosis of childhood TB can be established only by coupling clinical symptoms and laboratory tests [16].

In this study, the levels of urinary ESAT-6 were significantly different between TB and non-TB groups, either by microbiological and/or clinically confirmed as well as microbiological confirmation only. The study by Mukundan et al. in 2012 is the first study showing the direct detection of ESAT- 6 in serum samples obtained from patients with active TB. In these studies, examination of ESAT-6 (ELISA) conducted on 6 plasma samples in which three samples were samples obtained from active TB and 3 other samples were non-TB control and the results were obtained that ESAT- 6 was detected in three samples of active TB but not in non-TB control samples [11]. The study by Song et al. (2014) was a diagnostic test ESAT-6 (indirect ELISA) in meningitis TB, obtained sensitivity of $88 \%$ and a specificity of $92 \%$, there are significant differences between the mean levels of cerebrospinal fluid ESAT- 6 in patients with meningitis TB compare with non-meningitis TB. The ESAT-6 level was higher in meningitis TB than non-meningitis TB [12].

The sensitivity and specificity of urinary ESAT- 6 based on only microbiological confirmed was lower than the sensitivity and specificity urinary ESAT-6 based on microbiological and/or clinically confirmed. It may be caused by the difficulties in sampling of the children and paucibacillar conditions in children. In these cases, the diagnosis of TB can not use microbiologic examination only but also required clinical confirmation. This is supported by research Triasih and Graham (2011), which shows that the positive results of microbiological examination of sputum are only found in $10-15 \%$ of patients with suspected TB [5], and according to research by Jahromi (2014) positive cultures were obtained in $30-40 \%$ of cases [4]. Jahromi and Mood (2014) showed that sensitivity of culture examination is low in children because its general form is paucibacillary, di!culty to obtain sputum samples for children who can not cough up their sputum [4]. To overcome this, the selected samples from other locations were used such as culture of morning gastric lavage sample had a sensitivity of $30-40 \%$ while the nasopharyngeal aspiration was 24-30\%. Negative culture results cannot rule out the possibility of childhood TB. Other microscopic examination whose values were equal to culture examination on childhood TB is sputum smear examination, this examination has a sensitivity that varies between $25 \%$ and $75 \%$, but in children, sensitivity is only $<15 \%$. Most children with active TB are negative in smear examination and $10-15 \%$ of the children were shown to have smear-positive TB. Based on the data from the Indonesian Ministry of Health for TB Program in 2011, the percentage of smear-positive TB cases in children $0-14$ years is $6.3 \%$ of all TB cases children, this figure increased from 2010 at 5.3\% [2].

Until now, there were no studies that have been published regarding the diagnostic values of urinary ESAT-6 TB patient, especially in children. Urine samples have various advantages, especially in child whose sputum was paucibacillar, difficulty to expectorate the sputum, less invasive than blood or biopsy samples in extrapulmonary TB cases and can be used in cases of TB with HIV, whose sputum generally have fewer bacteria than in TB without HIV [6]. TB diagnosis in children has many flaws though using the gold standard. Until now, Indonesia using the TB scoring systemIDAI to diagnose TB in children. In other hand, there have been several studies that claim that this scoring system could cause inaccuracy TB diagnosis problems especially if there is history of household contact with TB patient. The study of Triasih and Graham state that the scoring system had a sensitivity of $47 \%$ and 
specificity of $68 \%$. These results indicate that the usage of TB scoring system to diagnose childhood TB need to be reobserved [5].

The TB diagnosis in this study did not use TB scoring system as defined by IDAI since 2008 but done by combining the clinical signs and symptoms and TB smear or culture. So that, the gold standard in this study more accurately described as reference standard. The reference standard used in this study was microbiological confirmed and/or clinically confirmed by competent pediatrician [17], [18].

ESAT- 6 is a small size, $6 \mathrm{kDa}$ protein and appears as a heterodimer in culture supernatants. ESAT-6 encoded by the region of difference 1 (RD1) of MTB which is an area that has consistently not found in attenuated or avirulen Mycobacterium bovis strains BCG. MTB uses the ESX-1 secretion system to deliver virulence proteins including ESAT- 6 during infection of host cells. ESAT- 6 forms a complex with culture filtrate protein-10 kDa (CFP-10) as a 1:1 ESAT-6:CFP-10 complex before exported out of the cell, ESAT- 6 can be separated from her partner CFP-10 at acidic $\mathrm{pH}$ (such as inside the phagosome). Individually, ESAT- 6 may cause membrane disruption and cytolysis [7]. ESAT-6 that is apart from CFP-10 will binds to liposomes containing dimyristoyl phosphatidylcholine and cholesterol (a component of mammals' cell membranes), causing instability and lysis of the liposomes. So that, ESAT-6 also plays a role in MTB mechanism to avoid the phagosome [19].

ESAT-6 size is small enough to allow its filtered unrestrictedly by the glomerulus so that can be detected in the urine. Kashino et al. in 2008 conducted a study of patients with active pulmonary TB which proved positive MTB culture patient after rule out any abnormalities or disorders of kidney and urinary tract as well as the possibility of renal TB. In such patients, TB antigen were detected in their urine which indicates that the TB antigens are derived from TB infection in the lungs [20].

\section{Study limitations}

The limitation of this study were the urine sample in this study were performed with random urine with various concentration of its analytes within it and it was difficult to obtained urine sample in pediatric patients, especially in child using diapers. Beside those, we did not examine urine of healthy control without TB.

\section{Conclusion}

We conclude that the levels of ESAT- 6 urine were higher in groups of patients with TB than non-TB.
The diagnostic value of urinary ESAT- 6 is moderate with sensitivity of $72 \%$ and specificity of $67 \%$. Urinary ESAT- 6 has good diagnostic value and could be considered of value when used in addition to microbiological tests and clinical examination.

\section{Acknowledgment}

We would like to thank to our colleagues from Department of Childhood, Faculty of Medicine Universitas Brawijaya/Saiful Anwar General Hospital, for the warmest cooperation. We also thank to Ministry of Research and Higher Education of Indonesia, and Dean of Medical Faculty of Universitas Brawijaya for supporting funding of the research.

\section{References}

1. Chakaya J, Khan M, Ntoumi F, Aklillu E, Fatima R, Mwaba P et al. Global tuberculosis report 2020-reflections on the global TB burden, treatment and prevention efforts. Int J Infect Dis. 2021;S1201-9712(21)00193-4. https://doi.org/10.1016/j. ijid.2021.02.107

2. Indonesian Ministry of Health. Technical Guidelines for Management of TB Children. Jakarta: Indonesian Ministry of Health; 2013.

3. Indonesia IDA. Diagnosis dan Tatalaksana Tuberkulosis Anak. Jakarta: Indonesian Ministry of Health; 2008.

4. Jahromi MK, Sharifi-Mood B. Pulmonary tuberculosis in children Int J Infect. 2014;1(3):e21116.

5. Triasih R, Graham SM. Limitations of the Indonesian pediatric tuberculosis scoring system in the context of child contact investigation. Paediatr Indones. 2011;51(6):332-7. https://doi. org/10.14238/pi51.6.2011.332-7

6. Tucci P, González-Sapienza G, Marin M. Pathogen-derived biomarkers for active tuberculosis diagnosis. Front Microbiol. 2014;5:549. https://doi.org/10.3389/fmicb.2014.00549 PMid:25368609

7. Maes RF. Was the WHO ban on TB serodiagnosis justified? Sci J Med Clin Trials. 2013;2013:292.

8. Ganguly N, Sharma P. Mycobacterium tuberculosis RD-1 Secreted Antigens as Protective and Risk Factors for Tuberculosis. Germany: BoD-Books on Demand; 2012. https:// doi.org/10.5772/30532

9. Holechek MJ. Glomerular filtration: An overview. Nephrol Nurs J. 2003;30(3):285.

PMid:12861896

10. Van Pinxteren LA, Ravn P, Agger EM, Pollock J, Andersen P. Diagnosis of tuberculosis based on the two specific antigens ESAT-6 and CFP10. Clin Diagn Lab Immunol. 2000;7(2):15560. https://doi.org/10.1128/cdli.7.2.155-160.2000 PMid:10702486

11. Mukundan H, Kumar S, Price DN, Ray SM, Lee YJ, Min S, et al. Rapid detection of Mycobacterium tuberculosis biomarkers in a sandwich immunoassay format using a waveguide-based optical biosensor. Tuberculosis. 2012;92(5):407-16. https://doi. 
org/10.1016/j.tube.2012.05.009

PMid:22710249

12. Song $F$, Sun $X$, Wang $X$, Nai $Y$, Liu Z. Early diagnosis of tuberculous meningitis by an indirect ELISA protocol based on the detection of the antigen ESAT- 6 in cerebrospinal fluid. Ir J Med Sci. 2014;183(1):85-8. https://doi.org/10.1007/ s11845-013-0980-4

PMid:23864405

13. Nayak S, Acharjya B. Mantoux test and its interpretation. Indian Dermatol Online J. 2012;3(1):2-6. https://doi. org/10.4103/2229-5178.93479

PMid:23130251

14. Product BL, Human M. Tuberculosis 6kDa/ESAT-6 ELISA Kit. Catalog No E3493Hu. Shanghai: Shanghai Crystal Day Biotech Co., Ltd.; 2016.

15. Chow SC. Sample Size and Determining Sample in Medical Research. $2^{\text {nd }}$ ed. Jakarta: Salemba Medika; 2009. p. 84-5.

16. López Ávalos GG, Prado Montes de Oca E. Classic and new diagnostic approaches to childhood tuberculosis. J Trop Med. 2012;2012:18219. https://doi.org/10.1155/2012/818219
PMid:22529869

17. World Health Organization. Global Tuberculosis Report 2013. Geneva: World Health Organization; 2013.

18. Wootton SH, Gonzalez BE, Pawlak R, Teeter LD, Smith KC, Musser JM, et al. Epidemiology of pediatric tuberculosis using traditional and molecular techniques: Houston, Texas. Pediatrics. 2005;116(5):1141-7. https://doi.org/10.1542/peds.2004-2701 PMid: 16264001

19. Ahmad S. Pathogenesis, immunology, and diagnosis of latent Mycobacterium tuberculosis infection. Clin Dev Immunol. 2011;2011:814943.

PMid:21234341

20. Kashino S, Pollock N, Napolitano D, Rodrigues V Jr., Campos-Neto A. Identification and characterization of Mycobacterium tuberculosis antigens in urine of patients with active pulmonary tuberculosis: An innovative and alternative approach of antigen discovery of useful microbial molecules. Clin Exp Immunol. 2008;153(1):56-62. https://doi. org/10.1111/j.1365-2249.2008.03672.x

PMid:18460016 